\title{
Discinesia ciliar primária: quando o pediatra deve suspeitar e como diagnosticar?
}

\author{
Primary ciliary dyskinesia: when the pediatrician must suspect and how to do the diagnosis?
}

Mary Anne K. Olm¹, Fabíola Villac Adde ${ }^{2}$, Luiz Vicente F. da Silva Filho ${ }^{3}$, Joaquim Carlos Rodrigues ${ }^{4}$

\section{RESUMO}

Objetivo: Revisar a discinesia ciliar primária (DCP) quanto aos seus aspectos ultra-estruturais, discriminar os defeitos ciliares primários dos secundários, descrever o quadro clínico, os testes laboratoriais de triagem e de diagnóstico disponíveis, bem como seu manejo clínico.

Fontes de dados: Pesquisa nas bases de dados Medline, Lilacs e SciELO, no período de 1980 a 2007.

Síntese dos dados: A DCP é uma doença autossômica recessiva que compromete a estrutura e/ou a função ciliar e, conseqüentemente, o transporte mucociliar. As manifestações clínicas envolvem o trato respiratório superior e inferior, com infecções recorrentes do ouvido médio, seios paranasais e pulmonares, que podem evoluir para bronquiectasias. Outras manifestações incluem situs inversus totalis e infertilidade masculina. $\mathrm{O}$ diagnóstico deve ser suspeitado pelos pediatras em várias situações: recém-nascidos de termo com desconforto respiratório sem causa aparente; neonatos portadores de dextrocardia; lactentes com tosse persistente e/ou infecções otorrinolaringológicas de repetição, excluindo-se as imunodeficiências e a fibrose cística; crianças com asma atípica e as com bronquiectasias sem causa definida. Os testes de triagem diagnóstica são os da sacarina e do óxido nítrico nasal. As avaliações do defeito ultra-estrutural e funcional exigem análise por microscopia eletrônica e da freqüência e formato da onda de batimento ciliar.

Conclusões: A DCP, apesar da baixa prevalência, é pouco diagnosticada pelas dificuldades de estabelecer o diagnóstico definitivo do defeito ciliar devido à complexidade da investigação laboratorial e pela falta de reconhecimento da doença pelos médicos. A suspeita clínica e o diagnóstico precoce são fundamentais para reduzir a morbidade e prevenir o desenvolvimento de complicações.

Palavras-chave: transtornos da motilidade ciliar; síndrome de Kartagener; bronquiectasia; infertilidade.

'Mestre em Medicina pela Universidade Federal de São Paulo e pós-graduando em nível de doutorado pela Faculdade de Medicina da Universidade de São Paulo (FMUSP), São Paulo, SP, Brasil 2Doutora em Medicina pela FMUSP e médica assistente da Unidade de Pneumologia Pediátrica do Instituto da Criança (ICr) do Hospital das Clínicas (HC) da FMUSP, São Paulo, SP, Brasil

${ }^{3}$ Doutor em Medicina pela FMUSP, médico assistente da Unidade de Pneumologia Pediátrica do ICr/HC-FMUSP, São Paulo, SP, Brasil

${ }^{4}$ Doutor em Medicina pela FMUSP, chefe da Unidade de Pneumologia Pediátrica do ICr/HC-FMUSP, São Paulo, SP, Brasil

\section{ABSTRACT}

Objective: To review primary ciliary dyskinesia (PCD) and its ultrastructural aspects, to differentiate primary from secondary ciliary defects and to describe the clinical features, screening and diagnostic laboratorial tests, and the clinical management of this disorder.

Data sources: A bibliographical search was obtained from Medline, Lilacs and SciELO databases, from 1980 to 2007.

Data synthesis: PCD is an autossomic recessive disorder with abnormal structure and/or function of the cilia, leading to reduced mucociliary clearance. The clinical manifestations include upper and lower respiratory tracts, with recurrent ear, sinus and lung infections that may progress to bronchiectasis. Situs inversus and male infertility are other clinical features of this disorder. PCD should be suspected by pediatricians in the following clinical situations: full term neonates with respiratory distress without apparent causes, presence of dextrocardia, infants with chronic cough and/or recurrent upper airways infections in the absence of immunodeficiency and cystic fibrosis, children with atypical asthma and bronchiectasis without a definitive cause. The diagnostic screening tests are the saccharine and nasal nitric oxide tests. Functional and ultrastructural evaluations demand an electronic microscopic analysis and the observation of the frequency and the pattern of the ciliary movement.

Conclusions: Although the prevalence of PCD is low, the difficulties in establishing the diagnosis due to the complex investigations demanded and the unfamiliarity of the disease by physicians lead to underdiagnosis. Early diagnosis and treatment of PCD are essential to reduce the morbidity and to avoid complications.

Key-words: ciliary motility disorders; Kartagener syndrome; bronchiectasis; infertility.

Endereço para correspondência

Joaquim Carlos Rodrigues

Unidade de Pneumologia Pediátrica do ICr/HC-FMUSP

Avenida Dr. Enéas de Carvalho Aguiar, 647

CEP 05403-900 - São Paulo/SP

E-mail: jocarod@uol.com.br

Recebido em:14/5/2007

Aprovado em: 1/8/2007 


\section{Introdução}

Várias crianças portadoras de infecções respiratórias recorrentes do trato respiratório superior e inferior e de pneumopatias crônicas, acompanhadas em ambulatórios especializados, persistem sem diagnóstico etiopatogênico, mesmo após a exclusão adequada das principais causas, tais como imunodeficiências, fibrose cística, síndrome aspirativa (por doença do refluxo gastroesofágico ou incoordenação motora da deglutição), bronquiolite obliterante, deficiência de alfa-1-antitripsina, etc. Nessa situação, uma das doenças pouco lembradas é a discinesia ciliar, cujo diagnóstico específico é difícil e depende de técnicas de investigação da ultraestrutura ciliar e dos movimentos ciliares.

As alterações genéticas da discinesia ciliar primária (DCP) afetam a formação de proteínas importantes que compõem e coordenam a estrutura e o movimento ciliar do epitélio respiratório e, conseqüientemente, o transporte mucociliar. O resultado é uma doença crônica obstrutiva, com envolvimento progressivo do trato respiratório superior e inferior, caracterizada por infecções recorrentes pulmonares, do ouvido médio e dos seios paranasais, que podem evoluir com o desenvolvimento de bronquiectasias. Outras manifestações incluem situs inversus totalis e infertilidade masculina. O diagnóstico ultra-estrutural é pouco acessível na prática clínica; porém, a utilização de alguns métodos de triagem simples e de fácil realização, tais como o teste da sacarina, podem ser úteis no diagnóstico presuntivo.



Figura 1 - Estrutura ciliar em corte axial (modificado de Cowan MJ et $a^{\left({ }^{(7)}\right)}$
O objetivo desse artigo foi fazer uma revisão atualizada da literatura, com base nos bancos de dados Medline, Lilacs e SciELO, no período de 1980 a 2007, discutindo os principais aspectos relacionados a apresentação, quadro clínico, testes de triagem, diagnóstico ultra-estrutural e manejo da DCP, bem como alertar os pediatras quanto à importância de considerar essa doença no diagnóstico diferencial das causas de base das afecções otorrinolaringológicas e/ou pulmonares crônicas e de repetição.

\section{Definição}

A discinesia ciliar é o nome genérico atribuído às doenças respiratórias nas quais ocorrem alterações estruturais e/ou disfunção ciliar, com conseqüente dano no transporte mucociliar, resultando em doenças oto-sinopulmonares ${ }^{(1)}$. Estas alterações podem ser primárias (congênitas ou geneticamente determinadas) ou secundárias, decorrentes de agressões externas ao epitélio respiratório. Estima-se incidência aproximada da DCP de 1/10.000 a 1/20.000, sendo mais freqüente em crianças com malformações cardíacas ou dextrocardia e anomalias do tubo digestivo.

A DCP é uma desordem autossômica recessiva. Análises de rastreamento genético feita em afetados e em seus familiares referem vários loci potenciais de localização nos cromossomos 3p, 4q, 5p, 7p, 10p, 11q, 13q, 15q, 16p, 17q e 19q q $^{(2)}$. Análises de familiares com deficiência específica de braços de dineína mostram alterações nos cromossomos 8q e 16p e, nos familiares com situs inversus, alterações nos cromossomas $8 \mathrm{q}$ e $19 \mathrm{q}^{(3,4)}$. A doença é muito heterogênea; os 200 tipos de proteína existentes no cílio dificultam os estudos de localização dos defeitos genéticos, por isso alguns genes isolados representam pequena parcela da casuística de pacientes afetados ${ }^{(4,5)}$.

\section{A estrutura e a função dos cílios respiratórios}

Os cílios normais dispõem-se à semelhança dos pêlos de um pincel e estão presos aos corpos basais em fileira, logo abaixo da membrana celular ${ }^{(6)}$. A estrutura ciliar em corte axial (Figura 1) apresenta ${ }^{(6,7)}$ :

1. nove pares de microtúbulos periféricos. Os elementos subfibra (A) e subfibra (B) de cada díade estão unidos entre si por vínculos de uma proteína conhecida como nexina. No microtúbulo (A) estão os braços de dineína externos e internos;

2. dois microtúbulos centrais ligados por uma ponte e circundados por uma membrana (bainha central);

3. espículas estriadas que se conectam dos túbulos centrais para os periféricos. 
As alterações ultra-estruturais na DCP podem incluir ${ }^{(6,8-11)}$ :

- defeitos nos braços de dineína: ausência dos braços internos e/ou externos de dineína, redução dos braços de dineína pela metade, braços de dineína curtos e retos em vez de curvos;

- ausência de espículas estriadas e bainha central;

- microtúbulos centrais ausentes, substituídos por um microtúbulo externo;

- defeitos das ligações de nexina, causando desarranjo dos microtúbulos;

- microtúbulos centrais e bainha central ausente;

- cílios de tamanho duplicado;

- corpos basais ausentes ou com redução do número de cílios;

- cílios com estrutura normal, porém imóveis.

A discinesia ciliar secundária ou adquirida pode resultar de agressões físicas e químicas ambientais (poluentes ambientais, fumaça de cigarro, álcool, exposição ao frio, etc.), que reduzem e prejudicam a atividade ciliar ${ }^{(12)}$. As infecções virais, bacterianas ou por outros agentes, como Mycoplasma pneumoniae, Chlamydia pneumoniae e Legionella pneumophila podem causar alterações estruturais persistentes ou transitórias no epitélio ciliar (descamação epitelial, desnudamento de cílios, desorganização e perda da membrana dos axonemas, formando cílios compostos, tubulopatias periféricas e centrais, além de encurtamento dos braços de dineína $)^{(12,13)}$. As anormalidades estruturais secundárias podem ocorrer em até $4 \%$ dos cílios analisados de indivíduos não portadores de discinesia ${ }^{(12,14)}$.

\section{Manisfestações clínicas}

O transporte mucociliar constitui um dos mais importantes mecanismos de defesa das vias aéreas. Prejuízos na depuração mucociliar aumentam o risco de infecções e de inflamação no sistema respiratório $^{(15)}$. O diagnóstico requer alto índice de suspeição clínica pelos pediatras, desde o período neonatal.

O diagnóstico clínico de discinesia ciliar deve ser considerado em uma série de circunstâncias ${ }^{(16-20)}$ :

- No período neonatal: presença de taquipnéia em recém-nascido de termo sem causa aparente, pneumonia inexplicada (em neonato sem fator de risco para infecção congênita) ${ }^{(21)}$, rinite, dextrocardia, situs inversus, doença cardíaca complexa (desordens de lateralidade), atresia de esôfago ou outros defeitos graves da função esofágica, atresia biliar ${ }^{(22)}$, hidrocefalia ${ }^{(23)}$ e história familiar positiva ${ }^{(3,8,18,24-27)}$.

- Em lactentes e crianças mais velhas: tosse produtiva, asma atípica ou de difícil controle, infecções pulmonares recorrentes, rinossinusites e/ou otites secretoras crônicas, pólipos nasais, bronquiectasias e perda auditiva ${ }^{(8,26,28)}$;
- Em adultos: infertilidade feminina ou masculina ${ }^{(29)}$, com espermatozóides imóveis ou de reduzida mobilidade, apesar da presença de fertilidade não excluir o diagnóstico em homens ${ }^{(8,26)}$.

A síndrome de Kartagener é uma malformação congênita rara, com incidência aproximada de um para 40.000 nascimentos ${ }^{(30)}$, caracterizada pela tríade: situs inversus, bronquiectasias e sinusopatia crônica ${ }^{(25,26)}$. O situs inversus costuma ocorrer em cerca de $50 \%$ dos pacientes com discinesia ciliar ${ }^{(28)}$. A coexistência de discinesia ciliar e do situs inversus relaciona-se à hipótese de que o cílio epitelial, na embriogênese precoce normal, possui posição e direção de batimento fixa e que o seu batimento determina a situação visceral $^{(30,31)}$. Quando os cílios são imóveis ou discinéticos, ocorre assimetria corporal ao acaso, resultando em casos variados de situs inversus ${ }^{(31)}$. As lesões ultra-estruturais dos cílios e flagelos dos pacientes com Kartagener variam entre os indivíduos e podem não ser iguais em um mesmo paciente, o que sugere que os axonemas dos cílios e flagelos devam ser controlados por genes comuns e genes diferentes $^{(32)}$. A síndrome de Kartagener é uma forma grave de discinesia ciliar, com imotilidade ciliar e de flagelos, o que determina infecções respiratórias crônicas e de repetição de caráter supurativo e infertilidade(33). As bronquiectasias, geralmente bilaterais e principalmente basais, decorrem da alteração no transporte mucociliar, com estase de secreções brônquicas, o que ocasiona infecção e inflamação nas vias aéreas e, conseqüentemente, lesão e destruição da cartilagem brônquica ${ }^{(34)}$. As Figuras 2 e 3 mostram os aspectos radiológicos e tomográficos do tórax nessa síndrome.

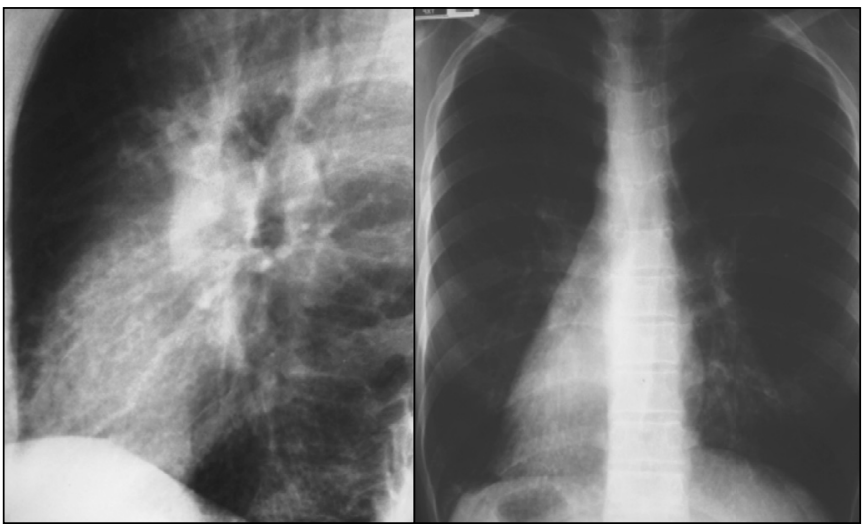

Figura 2 - Radiografias de tórax (perfil e póstero-anterior) de paciente com síndrome de Kartagener: pulmões hiperinsuflados e hipertransparentes. Opacidades lineares e arredondadas distribuídas pelo parênquima pulmonar, obliterando os contornos dos vasos arteriais bem evidentes nas bases. Destroposição cardíaca e bolha gástrica situada à direita 




Figura 3 - Tomografia computadorizada em alta resolução de paciente com síndrome de Kartagener: imagens com padrão em "árvore em brotamento", brônquios espessados e ectasiados. Bronquioloectasias com impactações mucóides, configurando o aspecto de "árvore em brotamento". Bronquiectasias císticas, varicosas e lineares no lobo médio

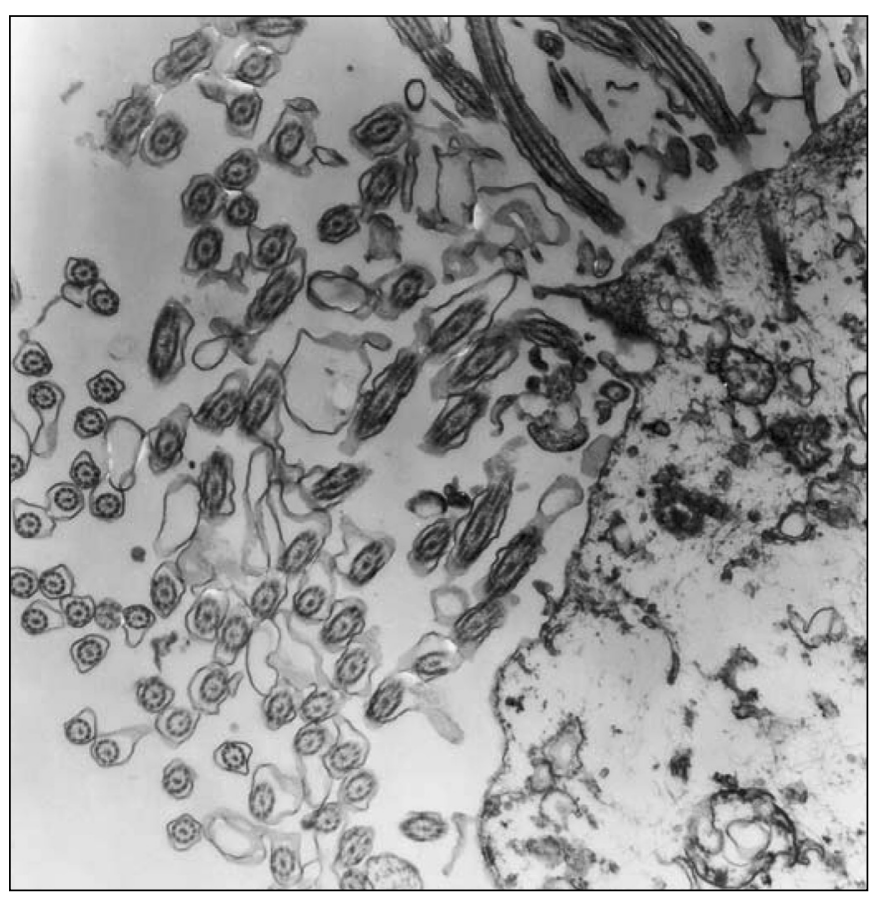

Figura 4 - Imagem obtida por microscopia eletrônica a partir de um escovado nasal

\section{Diagnóstico laboratorial}

O teste de triagem mais simples para o diagnóstico de DCP é o da sacarina, que, por ser colaborativo, só pode ser efetuado em crianças maiores de cinco anos ${ }^{(35)}$. O teste é um método simples, reprodutível, de baixo custo e avalia o transporte mucociliar da mucosa nasofaríngea ${ }^{(35,36)}$. O teste consiste na colocação de uma partícula de sacarina de $1 \mathrm{~mm}$ de diâmetro no assoalho da fossa nasal, cerca de $1 \mathrm{~cm}$ para dentro do corneto inferior. Mede-se o tempo em minutos entre a colocação da sacarina e o início da sensação de gosto doce na faringe. O teste é considerado alterado quando esse tempo for maior que 30 minutos, selecionando dessa forma os casos que merecem um estudo funcional e estrutural mais detalhado da estrutura ciliar.

Um teste de triagem mais sofisticado é a determinação da concentração do óxido nítrico (NO) nasal expirado. O NO é produzido no trato respiratório superior e inferior da via aérea humana e exerce muitas funções na defesa do hospedeiro, na atividade ciliar e na inflamação ${ }^{(37)}$. As suas concentrações nas vias aéreas superiores de indivíduos normais variam de 200 a 2.000 partes por bilhão (ppb), sendo muito mais elevada do que os níveis observados no trato respiratório inferior $(4-160 \mathrm{ppb})^{(37)}$. Nos pacientes com DCP, as concentrações de NO exaladas da região naso-sinusal são muito baixas (menores que $200 \mathrm{ppb})^{(38)}$, supostamente por incapacidade genética de produzi-lo. Atualmente, existem recomendações internacionais para a mensuração do NO nasal expirado ${ }^{(39)}$. O NO pode ser coletado por um probe nasal (medida on line) ou por sacos coletores (medida off-line) e as concentrações são determinadas por equipamentos analisadores de NO. Estes equipamentos são de alto custo, porém, quando disponíveis, a determinação de valores de NO nasal abaixo de 250ppm pode constituir um método útil para a triagem da doença ${ }^{(38,40)}$.

Os pacientes com teste da sacarina alterado (maior que 30 minutos) ou teste do NO nasal alterado (menor que 250ppb) devem ser submetidos ao estudo da freqüência do batimento ciliar, do padrão de onda ciliar e da ultra-estrutura dos cílios. O material para análise detalhada da ultra-estrutura e função ciliar pode ser obtido por biópsia da mucosa nasal ou por escovado nasal. Este último é de mais fácil realização, tem menor custo e morbidade e permite obter material para a observação in vivo da atividade ciliar ${ }^{(41-45)}$.

A amostra obtida do epitélio nasal deve ser encaminhada imediatamente ao laboratório para determinar a freqüência de batimento ciliar, verificar o padrão de movimento ciliar e para a análise da ultra-estrutura ciliar por microscopia eletrô- 
nica (Figura 4), com a finalidade de avaliar o tipo de defeito existente. Considera-se que existe discinesia ciliar quando a freqüência dos batimentos ciliares for menor do que $11 \mathrm{Hertz}$ (11 ciclos/segundo) $)^{(46)}$. No entanto, a interpretação da freqüência de batimento ciliar deve ser acompanhada pela análise do padrão de batimento (formato de onda ciliar), pois cerca de $10 \%$ dos casos de freqüência de batimento ciliar considerados normais por vídeos de alta velocidade podem ter alteração no seu padrão de batimento ${ }^{(36)}$. Em centros de investigação ultra-especializados, pode-se realizar a cultura de células ciliares com o objetivo de discriminar os defeitos primários dos secundários ${ }^{(47-52)}$.

\section{Principais complicações}

Em virtude das dificuldades relacionadas aos recursos laboratoriais, as crianças com discinesia ciliar são subdiagnosticadas ou têm o diagnóstico tardio. Estima-se que ocorra perda de função pulmonar, baseada no VEF1, de cerca de $0,8 \%$ ao ano ${ }^{(33,53)}$. As bronquiectasias aparecem no decorrer da idade, em conseqüência às infecções pulmonares repetidas e à inflamação pulmonar crônica. Em casuística de 72 pacientes, 98\% daqueles acima de 18 anos apresentavam evidências tomográficas e sintomas de bronquiectasias, comparados a $61 \%$ dos menores de 18 anos $^{(33)}$. Os principais agentes microbiológicos isolados do escarro ou de material de orofaringe desses pacientes são: Haemophilus influenzae, Streptococcus pneumoniae, Staphylococcus aureus e Pseudomonas aeruginosa, sendo esta mais freqüente nos pacientes com doença avançada ${ }^{(26,33,53)}$.

\section{Manejo, evolução e prognóstico}

O tratamento consiste em antibioticoterapia nas exacerbações respiratórias, utilizando, sempre que possível, fármacos com ação contra os agentes prevalentes, além de basear a escolha do antibiótico em culturas e sensibilidade antimicrobiana, quando possível. Nos portadores de bronquiectasias de caráter supurativo, deve-se orientar a antibioticoterapia profilática e a fisioterapia respiratória continua. É importante educar pacientes e familiares para evitar alérgenos ambientais e fumo, além de estimular a prática de exercícios físicos ${ }^{(36,54,55)}$.

\section{Referências bibliográficas}

1. Eley L, Yates LM, Goodship JA. Cilia and disease. Curr Opin Genet Dev 2005;15:308-14.

2. Bush A, Ferkol T. Movement: the emerging genetics of primary ciliary dyskinesia. Am J Respir Crit Care Med 2006 15;174:109-10.

3. Van's Gravesande KS, Omran H. Primary ciliary dyskinesia: clinical presentation, diagnosis and genetics. Ann Med 2005;37:439-49.
Deve-se recomendar a vacinação pneumocócica e a imunização anual contra a gripe. A discinesia ciliar, apesar da alta morbidade, tem baixa mortalidade ${ }^{(36)}$. Os pacientes portadores de bronquiectasias, com doença supurativa crônica, podem ter comprometimento importante da sua qualidade de vida. Nesta situação, tratando-se de bronquiectasias localizadas e quando todos os recursos clínicos se esgotaram, excepcionalmente indicam-se ressecções cirúrgicas lobares ${ }^{(56)}$.

\section{Conclusões e perspectivas}

A DCP é uma doença subdiagnosticada. Os pediatras devem estar alertas para sua suspeita, principalmente nas seguintes situações:

1. crianças com doença compatível com DCP sem uma causa definida, tal como fibrose cística ou imunodeficiência;

2. desconforto respiratório em neonatos de termo;

3. bronquiectasias e/ou doença supurativa das vias aéreas de etiologia desconhecida, mesmo sem situs inversus;

4. tosse crônica persistente;

5. sinusite crônica ou recorrente;

6. otite média crônica ou otites graves recorrentes;

7. situs inversus com ou sem cardiopatia congênita associada;

8. história familiar de DCP.

O diagnóstico deve ser feito com base nos critérios clínicos, em testes de triagem - teste da sacarina e do NO, quando disponível - e em laboratórios especializados na análise ultra-estrutural e funcional dos cílios, preferencialmente de material obtido por escovado nasal. A DCP é uma doença geneticamente heterogênea, com o envolvimento de múltiplos loci cromossômicos ${ }^{(57)}$. Há perspectivas atuais para a detecção da anomalia genética. Duas mutações gênicas que controlam a estrutura dos braços externos de dineína (DNAI1 e DNAH5) são responsáveis por 10 e $28 \%$ das mutações descritas, respectivamente ${ }^{(58)}$. Atualmente, estão disponíveis testes de biologia molecular capazes de detectar tais mutações por meio do seqüenciação de regiões codificantes (exons) e de regiões próximas não codificantes (íntrons) dos genes DNAI1 (exons

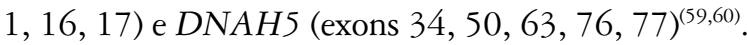

4. Guichard C, Harricane MC, Lafitte JJ, Godard P, Zaegel M, Tack V et al. Axonemal dynein intermediate-chain gene (DNAl1) mutations result in situs inversus and primary ciliary dyskinesia (Kartagener syndrome). Am J Hum Genet 2001;68:1030-5.

5. Blouin JL, Meeks M, Radhakrishna U, Sainsbury A, Gehring C, Saïl GD et al. Primary ciliary dyskinesia: a genome-wide linkage analysis reveals extensive locus heterogeneity. Eur J Hum Genet 2000;8:109-18. 
6. Felix $\mathrm{H}$, Holzmann D. Function and ultrastructure of cilia in primary ciliary dyskinesia. Schweiz Med Wochenschr 2000;130:699-704.

7. Cowan MJ, Gladwin MT, Shelhamer JH. Disorders of ciliary motility. Am J Med Sci 2001;321:3-10.

8. Afzelius BA. Ciliary structure in health and disease. Acta Otorhinolaryngol Belg 2000;54:287-91.

9. Bush A. Primary ciliary dyskinesia. Acta Otorhinolaryngol Belg 2000;54:317-24.

10. Carlen B, Stenram U. Ultrastructural diagnosis in the immotile cilia syndrome. Ultrastruct Pathol 1987;11:653-8.

11. Barlocco EG, Valletta EA, Canciani M, Lungarella G, Gardi C, De Santi MM et al. Ultrastructural ciliary defects in children with recurrent infections of the lower respiratory tract. Pediatr Pulmonol 1991;10:11-7.

12. Bertrand $B$, Collet $S$, Eloy $P$, Rombaux $P$. Secondary ciliary dyskinesia in upper respiratory tract. Acta Otorhinolaryngol Belg 2000;54:309-16.

13. Chilvers MA, McKean M, Rutman A, Myint BS, Silverman M, O'Callaghan C. The effects of coronavirus on human nasal ciliated respiratory epithelium. Eur Respir J 2001;18:965-70.

14. Rossman CM, Lee RM, Forrest JB, Newhouse MT. Nasal ciliary ultrastructure and function in patients with primary ciliary dyskinesia compared with that in normal subjects and in subjects with various respiratory diseases. Am Rev Respir Dis 1984;129:161-7.

15. Willems T, Jorissen M. Correlations between ciliary structure and ciliary function. Acta Otorhinolaryngol Belg 2000;54:299-308.

16. Bush A, Cole P, Hariri M, Mackay I, Phillips G, O'Callaghan C et al. Primary ciliary dyskinesia: diagnosis and standards of care. Eur Respir $\mathrm{J}$ 1998;12:982-8.

17. Coren ME, Meeks M, Morrison I, BuchdahI RM, Bush A. Primary ciliary dyskinesia: age at diagnosis and symptom history. Acta Paediatr 2002;91:667-9.

18. Holzmann D, Ott PM, Felix H. Diagnostic approach to primary ciliary dyskinesia: a review. Eur J Pediatr 2000;159:95-8.

19. Chin GY, Karas DE, Kashgarian M. Correlation of presentation and pathologic condition in primary ciliary dyskinesia. Arch Otolaryngol Head Neck Surg 2002;128:1292-4

20. Toledo MF, Adde FV. Primary ciliary dyskinesia in children. J Pediatr (Rio J) 2000;76:9-16

21. Holzmann $D$, Felix H. Neonatal respiratory distress syndrome--a sign of primary ciliary dyskinesia? Eur J Pediatr 2000;159:857-60.

22. Gershoni-Baruch R, Gottfried E, Pery M, Sahin A, Etzioni A. Immotile cilia syndrome including polysplenia, situs inversus, and extrahepatic biliary atresia. Am J Med Genet 1989;33:390-3.

23. Greenstone MA, Jones RW, Dewar A, Neville BG, Cole PJ. Hydrocephalus and primary ciliary dyskinesia. Arch Dis Child 1984;59:481-2.

24. Le Mauviel L. Primary ciliary dyskinesia. West J Med 1991;155:280-3.

25. Meeks M, Bush A. Primary ciliary dyskinesia (PCD). Pediatr Pulmonol 2000;29:307-16.

26. Rossman CM, Newhouse MT. Primary ciliary dyskinesia: evaluation and management. Pediatr Pulmonol 1988;5:36-50.

27. Engesaeth VG, Warner JO, Bush A. New associations of primary ciliary dyskinesia syndrome. Pediatr Pulmonol 1993;16:9-12.

28. Turner JA, Corkey CW, Lee JY, Levison H, Sturgess J. Clinical expressions of immotile cilia syndrome. Pediatrics 1981;67:805-10.

29. Afzelius BA, Eliasson R. Male and female infertility problems in the immotilecilia syndrome. Eur J Respir Dis Suppl 1983;127:144-7.

30. Afzelius BA. Genetics and pulmonary medicine. 6 . Immotile cilia syndrome: past, present, and prospects for the future. Thorax 1998;53:894-7.

31. Afzelius BA. Situs inversus and ciliary abnormalities. What is the connection? Int J Dev Biol 1995;39:839-44.

32. Escudier E, Escalier D, Pinchon MC, Boucherat M, Bernaudin JF, Fleury-Feith J. Dissimilar expression of axonemal anomalies in respiratory cilia and sperm flagella in infertile men. Am Rev Respir Dis 1990;142:674-9.

33. Noone PG, Leigh MW, Sannuti A, Minnix SL, Carson JL, Hazucha M et al. Primary ciliary dyskinesia: diagnostic and phenotypic features. Am J Respir Crit Care Med 2004;169:459-67.

34. de longh RU, Rutland J. Ciliary defects in healthy subjects, bronchiectasis, and primary ciliary dyskinesia. Am J Respir Crit Care Med 1995;151:1559-67.

35. Adde FV, Rozov T. Teste da sacarina em crianças. J Pneumol 1997;23:66-70.
36. Bush A, O'Callaghan C. Primary ciliary dyskinesia. Arch Dis Child 2002;87:363-5.

37. Lefevere L, Willems T, Lindberg S, Jorissen M. Nasal nitric oxide. Acta Otorhinolaryngol Belg 2000;54:271-80.

38. Karadag B, James AJ, Gültekin E, Wilson NM, Bush A. Nasal and lower airway level of nitric oxide in children with primary ciliary dyskinesia. Eur Respir $\mathrm{J}$ 1999;13:1402-5.

39. Kharitonov S, Alving K, Barnes PJ. Exhaled and nasal nitric oxide measurements: recommendations. The European Respiratory Society Task Force. Eur Respir J 1997;10:1683-93.

40. Narang I, Ersu R, Wilson NM, Bush A. Nitric oxide in chronic airway inflammation in children: diagnostic use and pathophysiological significance. Thorax 2002;57:586-9.

41. MacCormick J, Robb I, Kovesi T, Carpenter B. Optimal biopsy techniques in the diagnosis of primary ciliary dyskinesia. J Otolaryngol 2002;31:13-7.

42. Rutland J, Griffin W, Cole P. Nasal brushing and measurement of ciliary beat frequency. An in vitro method for evaluating pharmacologic effects on human cilia. Chest 1981;80(Suppl):865-7.

43. Rutland J, Dewar A, Cox T, Cole P. Nasal brushing for the study of ciliary ultrastructure. J Clin Pathol 1982;35:357-9.

44. Rutland J, Cole PJ. Non-invasive sampling of nasal cilia for measurement of beat frequency and study of ultrastructure. Lancet 1980;2:564-5.

45. Bush A, Pohunek P. Brush biopsy and mucosal biopsy. Am J Respir Crit Care Med 2000;162:S18-22.

46. Chilvers MA, Rutman A, O'Callaghan C. Ciliary beat pattern is associated with specific ultrastructural defects in primary ciliary dyskinesia. J Allergy Clin Immunol 2003;112:518-24.

47. Jorissen M, Willems T, Van der Schueren B. Ciliary function analysis for the diagnosis of primary ciliary dyskinesia: advantages of ciliogenesis in culture. Acta Otolaryngol 2000;120:291-5.

48. Jorissen M, Van der Schueren B, Van den Berghe H, Cassiman JJ. Ciliogenesis in cultured human nasal epithelium. ORL J Otorhinolaryngol Relat Spec 1990;52:368-74.

49. Jorissen M, Willems T, De Boeck K. Diagnostic evaluation of mucociliary transport: from symptoms to coordinated ciliary activity after ciliogenesis in culture. Am J Rhinol 2000;14:345-52.

50. Jorissen M, Willems T, Van der Schueren B, Verbeken E. Secondary ciliary dyskinesia is absent after ciliogenesis in culture. Acta Otorhinolaryngol Belg 2000;54:333-42.

51. Jorissen M, Willems $T$. Success rates of respiratory epithelial cell culture techniques with ciliogenesis for diagnosing primary ciliary dyskinesia. Acta Otorhinolaryngol Belg 2000;54:357-65.

52. Jorissen M, Willems T, Van der Schueren B, Verbeken E, De Boeck K. Ultrastructural expression of primary ciliary dyskinesia after ciliogenesis in culture. Acta Otorhinolaryngol Belg 2000;54:343-56.

53. Ellerman $A$, Bisgaard $H$. Longitudinal study of lung function in a cohort of primary ciliary dyskinesia. Eur Respir J 1997;10:2376-9.

54. Phillips GE, Thomas S, Heather S, Bush A. Airway response of children with primary ciliary dyskinesia to exercise and beta2-agonist challenge. Eur Respir J 1998;11:1389-91.

55. Koh YY, Park Y, Jeong JH, Kim CK, Min YG, Chi JG. The effect of regular salbutamol on lung function and bronchial responsiveness in patients with primary ciliary dyskinesia. Chest 2000;117:427-33.

56. Smit HJ, Schreurs AJ, Van den Bosch JM, Westermann CJ. Is resection of bronchiectasis beneficial in patients with primary ciliary dyskinesia? Chest 1996;109:1541-4.

57. Zariwala MA, Knowles MR, Omran H. Genetic defects in ciliary structure and function. Annu Rev Physiol 2007;69:423-50.

58. Noone PG, Zariwala M, Sannuti A, Minnix S, Leigh MW, Carson J et al. Mutations in DNAl1 (IC78) cause primary ciliary dyskinesia. Chest 2002;121(Suppl):97S.

59. Zariwala MA, Leigh MW, Ceppa F, Kennedy MP, Noone PG, Carson JL et al. Mutations of DNAl1 in primary ciliary dyskinesia: evidence of founder effect in a common mutation. Am J Respir Crit Care Med 2006;174:858-66.

60. Hornef N, Olbrich H, Horvath J, Zariwala MA, Fliegauf M, Loges NT et al. DNAH5 mutations are a common cause of primary ciliary dyskinesia with outer dynein arm defects. Am J Respir Crit Care Med 2006;174:120-6 